\title{
Pharmacometabolomics study identifies circulating spermidine and tryptophan as potential biomarkers associated with the complete pathological response to trastuzumab-paclitaxel neoadjuvant therapy in HER-2 positive breast cancer
}

\author{
Gianmaria Mioloㅅ, Elena Muraro ${ }^{2}$, Donatella Caruso ${ }^{3}$, Diana Crivellari ${ }^{1}$, Anthony Ash ${ }^{4}$, \\ Simona Scalone ${ }^{1}$, Davide Lombardi ${ }^{1}$, Flavio Rizzolio ${ }^{2}$, Antonio Giordano ${ }^{5}$, Giuseppe \\ Corona $^{2}$ \\ ${ }^{1}$ Department of Medical Oncology, IRCCS-National Cancer Institute, Aviano, Italy \\ ${ }^{2}$ Department of Translational Research, IRCCS-National Cancer Institute, Aviano, Italy \\ ${ }^{3}$ Department of Pharmacological and Bimolecular Science, University of Milan, Milan, Italy \\ ${ }^{4}$ Department of Biological Chemistry, Norwich Research Park, Norwich, United Kingdom \\ ${ }^{5}$ Sbarro Institute for Cancer Research and Molecular Medicine, Center for Biotechnology, College of Science and Technology, \\ Temple University, Philadelphia, PA, USA
}

Correspondence to: Giuseppe Corona, email: giuseppe.corona@cro.it

Keywords: pharmacometabolomics, pharmacometabonomics, metabolomics, breast, cancer

Received: October 30, $2015 \quad$ Accepted: April 28, $2016 \quad$ Published: May 19, 2016

\section{ABSTRACT}

Defining biomarkers that predict therapeutic effects and adverse events is a crucial mandate to guide patient selection for personalized cancer treatments. In the present study, we applied a pharmacometabolomics approach to identify biomarkers potentially associated with pathological complete response to trastuzumab-paclitaxel neoadjuvant therapy in HER-2 positive breast cancer patients. Based on histological response the 34 patients enrolled in the study were subdivided into two groups: good responders $(n=15)$ and poor responders $(n=19)$. The pre-treatment serum targeted metabolomics profile of all patients were analyzed by liquid chromatography tandem mass spectrometry and the differences in the metabolomics profile between the two groups was investigated by multivariate partial least squares discrimination analysis. The most relevant metabolites that differentiate the two groups of patients were spermidine and tryptophan. The Good responders showed higher levels of spermidine and lower amounts of tryptophan compared with the poor responders $(p<0.001$, $q<0.05$ ). The serum level of these two metabolites identified patients who achieved a pathological complete response with a sensitivity of $90 \%[0.79-1.00]$ and a specificity of $0.87 \%$ [0.67-1.00]. These preliminary results support the role played by the individual patients' metabolism in determining the response to cancer treatments and may be a useful tool to select patients that are more likely to benefit from the trastuzumab-paclitaxel treatment.

\section{INTRODUCTION}

The introduction of trastuzumab revolutionized the treatment of HER-2-positive breast cancer (BC) and now represents the gold standard drug used alongside other pharmacological regimens both in adjuvant and neoadjuvant settings [1-4]. The latter approach may be able to reduce the tumour mass of locally advanced BC, decrease the subclinical micro-metastatic disease and improve the rates of breast-conserving surgeries $[1,5]$. In this setting, the induction of a pathological complete response (pCR), i.e. the absence of cancer cells in breast tissue after therapy, is associated with improved disease free survival (DFS), overall survival (OS) and reduced relapse rates $[6,7]$. Even if the addition of trastuzumab strongly increased the $\mathrm{pCR}$ rates in HER-2 positive $\mathrm{BC}$ patients [8] there is still a significant subgroup of patients that does not benefit from this treatment [9]. For this 
reason, the identification of specific biomarkers that are able to predict an unfavourable response to trastuzumabbased neoadjuvant treatment could be clinically useful in order to select more effective treatments.

The survey for biomarkers, managed both through retrospective clinical analysis and gene expression studies [10] proved to be very challenging, showing that also the level of HER-2 expression, the most obvious candidate biomarker for HER-2 targeting therapy, resulted to be surprisingly ambiguous [11-13]. The multifaceted mode of action of trastuzumab may be responsible for the difficulty in identifying, predictive biomarkers since as monoclonal antibody trastuzumab acts also through immune-mediated mechanisms that involve host immune cells [14]. The required contribution of host effectors suggests a direct involvement of individuals' endogenous biochemical factors, which could thus represent an innovative and complementary front for predictive biomarkers research. Moreover, other recently proposed surrogate markers of response to neoadjuvant treatment are based on patients' characteristics such as the body mass index (BMI). Indeed, a higher BMI was found associated with lower rate of $\mathrm{pCR}$ and a detrimental impact on survival [15]. This contributes to retain that the host response to the tumour is perhaps equally important to determining the pharmacological effect, which can be highlighted by an individual's specific biochemical signature.

Pharmacometabolomics is an innovative "omic" methodology that is able to access patients' biochemical status and is therefore useful to understand the host's response to drug treatments. This new approach is based on the identification of individuals' metabolomic profiles, which represent a large repertoire of metabolites that may reflect the complex interactions among gene expression, protein expression, physio-pathological conditions, age, gut microbiome, and the environment better than other "omic" profiles. Consequently, the metabolomics profile is more closely associated to a patient's pharmacological phenotype and could be more informative than genomic and proteomic data clarifying better the mechanisms of inter-patient variability to drug therapy [16-19]. The potential for pharmacometabolomics has already been demonstrated for different classes of drugs in preclinical investigations, as well as in clinical pharmacological settings [20-25]. A few investigations have been applied to the pharmacological treatment of BCs. In particular, two previous investigations analysed the predictive value of basal serum metabolites on the outcome of BC patients in the metastatic and neoadjuvant settings [26, 27] but their results were contradictory. One of these studies consisted of a pilot multicentric investigation that attempted to correlate the serum metabolomics profile with patient outcome and treatments toxicity [26]. However, this study failed to identify any correlations between the patients' metabolomics profile and the therapies effect. More recently, a metabolomics investigation found that $\mathrm{pCR}$ correlated with changes in serum level of methionine, glutamine and linoleic acid [27]. However, the histological heterogeneity of the tumours and the different treatments administered limited the data interpretation for both these studies.

The present study was designed to explore the potential use of a pharmacometabolomics approach as a means to identify pre-treatment serum metabolite biomarkers associated with the $\mathrm{pCR}$ in a homogeneous monocenter cohort of HER-2 positive BC patients after neoadjuvant trastuzumab-paclitaxel treatment. We found that patients with lower serum concentrations of tryptophan and higher concentrations of spermidine responded more successfully to the treatment. This underlines the role that the individual's metabolic trait may have on determining the pharmacological outcome of cancer therapy.

\section{RESULTS}

\section{Patient characteristics}

The demographic and pathological characteristics of the patients investigated are reported in Table 1. Patients were divided into two groups according to their histological response to the trastuzumab-paclitaxel neoadjuvant treatment. The good responders (GR) $(n=15)$, who achieved pCR and the poor responders (PR) $(n=19)$, who achieved only a partial pathological response where residual disease was still revealed after the neoadjuvant treatment. The two groups did not differ significantly by age, BMI, tumour stage, grade and hormonal receptor expression.

In both groups approximately half of the patients had hormone receptor positive tumours. Although not statistically significant at diagnosis, the GR group was characterized by a higher frequency of stage III tumours, which is generally indicative of a more aggressive phenotype with a relatively poor prognosis.

\section{Metabolomics data analysis and biomarker identification}

The pre-treatment serum targeted metabolomics profile data, determined by a validated LC-MS/MS, were analysed using supervisor partial least squares discrimination analysis (PLS-DA). This analysis was carried out to investigate if differences in the quantitative metabolomics profiles of the patients were able to distinguish the GR from the PR group. The results of this multi-parametric approach are summarized in the PLS-DA graph (Figure 1), where each point corresponds to the metabolite profile of each patient. From the quantitative metabolomics data, the GR group had a spatial distribution that was significantly different from that of PR group. The PSA-DA model was further refined to eliminate potential noise by focusing on the metabolites that showed 
Table 1: Patients' characteristics

\begin{tabular}{|c|c|c|c|}
\hline Parameters & $G R$ & $P R$ & $p$ \\
\hline \multicolumn{4}{|l|}{ Patients } \\
\hline 34 & 15 & 19 & \\
\hline Age (years) & & & $\mathrm{NS}^{\#}$ \\
\hline Median (range) & $42(28-58)$ & $49(23-70)$ & \\
\hline BMI (Kg/m2) & & & $\mathrm{NS}^{\#}$ \\
\hline Mean \pm SD & $24.1 \pm 4.9$ & $25.8 \pm 5.6$ & \\
\hline Stage & & & $\mathrm{NS}^{\#}$ \\
\hline IIA & 2 & 1 & \\
\hline IIB & 8 & 16 & \\
\hline IIIA & 5 & 2 & \\
\hline Grade & & & $\mathrm{NS}^{\S}$ \\
\hline G2 & 2 & 3 & \\
\hline G3 & 9 & 15 & \\
\hline Gx & 4 & 1 & \\
\hline $\mathrm{ER} / \mathrm{PgR}$ & & & $\mathrm{NS}^{\S}$ \\
\hline neg/neg & 7 & 8 & \\
\hline pos/neg & 4 & 3 & \\
\hline pos/pos & 4 & 7 & \\
\hline neg/pos & 0 & 1 & \\
\hline
\end{tabular}

"unpaired $t$-Test, ${ }^{\S}$ Chi-Square test. BMI Body mass index, ER:Estrogen receptors, PgR:

progesterone receptors.

a significant difference among the two groups of patients. The internal cross validation of the refined PLS-DA model used in this study demonstrated good modelling and predictive capabilities $\left(85 \%\right.$ accuracy, good $\mathrm{R}^{2}(0.73)$ and $\mathrm{Q}^{2}(0.57)$. The values and permutation testing did not reveal any significant $(p<0.003)$ potential for over-fitting the model.

In order to select the metabolites that showed a significant difference between the GR and PR metabolomic profiles a variable influence on the projection (VIP) parameter was used. The most relevant metabolites (VIP > 1) were: spermidine (Spd), tryptophan (Trp), propylcarnitine (C3) and the two phosphatidylcholine diacyl phospholipids (PC aa) $\mathrm{PC}$ aa $\mathrm{C} 26: 0$ and $\mathrm{PC}$ aa $\mathrm{C} 30: 2$ (Figure 2). The relative concentration distributions of such metabolites between the two groups of patients can be visualized in a heatmap (Figure 3). Among the five relevant metabolites (Spd; Trp; C3; PCaa C26:0 and PC aa $\mathrm{C} 30: 2)$, the difference in Spd concentration levels resulted the most clearly differentiated between the two groups. Furthermore, when an alternative statistical analysis was performed such as the Significance Analysis of Microarray
(SAM) to account for potential False Discovery Rates (FDR), only Spd and Trp showed significant differences between the two groups of patients (Figure 4). The mean $\pm \mathrm{SD}$ serum concentration of bioactive amine Spd in the GR group was significantly higher, $0.15 \pm 0.06 \mu \mathrm{M}$ vs $0.09 \pm 0.032 \mu \mathrm{M}(p<0.001, q<0.05)$, relative to the PR group. Whilst, the level of Trp was significantly lower in the GR group $61.19 \pm 8.46 \mu \mathrm{M} v s 73.82 \pm 9.23 \mu \mathrm{M}$ ( $p=0.001, q<0.05)$ relative to the PR group. Consequently, the anabolic and catabolic routes involved in the metabolism of Spd and Trp between the two groups of patients was further investigated. This analysis was carried out using the available quantitative metabolomics profile data, to explore any other associated metabolic alterations that could be potentially useful in the development of a predictive model for $\mathrm{pCR}$ after neoadjuvant trastuzumab-paclitaxel treatment.

\section{Spermidine and tryptophan metabolic pathways}

The Spd is a metabolite derived from the catabolic process of ornitine (Orn) to putrescine (Put) that requires methionine (Met) activation for its synthesis (Figure 5). 
The quantitative data from the metabolomics profile indicated that between the GR and PR groups there were no significant differences in the serum levels of the Orn and Met precursors: $81 \pm 16 \mu \mathrm{M} v s 87 \pm 22 \mu \mathrm{M}$ for Orn and $18.87 \pm 4.08 \mu \mathrm{M} v s 21 \pm 5 \mu \mathrm{M}$ for Met, respectively. In addition, the serum level of Put showed no significant differences between the two groups of patients: GR $0.14 \pm$ $0.05 \mu \mathrm{M}$ vs PR $0.13 \pm 0.05 \mu \mathrm{M}$. Spermine, which is also involved in the pathway of bio-amine synthesis, was lower than the limit of detection for all patients investigated. The GR group characterized by a higher concentration of Spd as compared with the PR group, showed a significantly lower Orn/Spd ratio: $610 \pm 205$ vs $1107 \pm 534(p<0.05)$ and a significantly lower Put/Spd ratio $1.0 \pm 0.4$ vs $1.6 \pm 0.8$ $(p<0.05)$.

The Trp represents an essential amino acid that provides a building block to a vast array of proteins. It also acts as biochemical precursor for the synthesis of niacin cofactor, through pathways of kynurenine (Kyn) and for the synthesis of melatonin from serotonin (Set) via the methoxyindoles pathway (Figure 6). Within the Kyn pathway, the low serum level of Trp observed in the GR group was associated with a significantly $(p<0.05)$ lower level of the intermediate Kyn, $2.2 \pm 0.4$ vs $2.8 \pm 0.7 \mu \mathrm{M}$, compared to the PR group. However, the ratio between Trp and Kyn showed no significant differences between two groups ( $27 \pm 4$ vs $28 \pm 7$ ). In addition, the mean serum concentrations of Set (via the methoxyindoles pathway) also showed no significant differences between the two groups, GR $0.60 \pm 0.16 \mu \mathrm{M} v s$ PR $0.55 \pm 0.27 \mu \mathrm{M}$ while the mean value of the Trp/Set ratio was significantly lower in the GR group compared with the PR group: $108 \pm 29$ vs $159 \pm 70,(p<0.05)$ (Figure 6).

\section{Receiver operating characteristic curve}

To demonstrate the diagnostic value of the two most relevant serum metabolites, a receiver operating characteristic (ROC) curve was applied to the data. This helped to evaluate the predictive power of the two metabolites in identifying patients that would best benefit from the neoadjuvant treatment. The ROC curves were produced using the serum concentration of Spd and Trp both independently and combined. The ROC curve data was

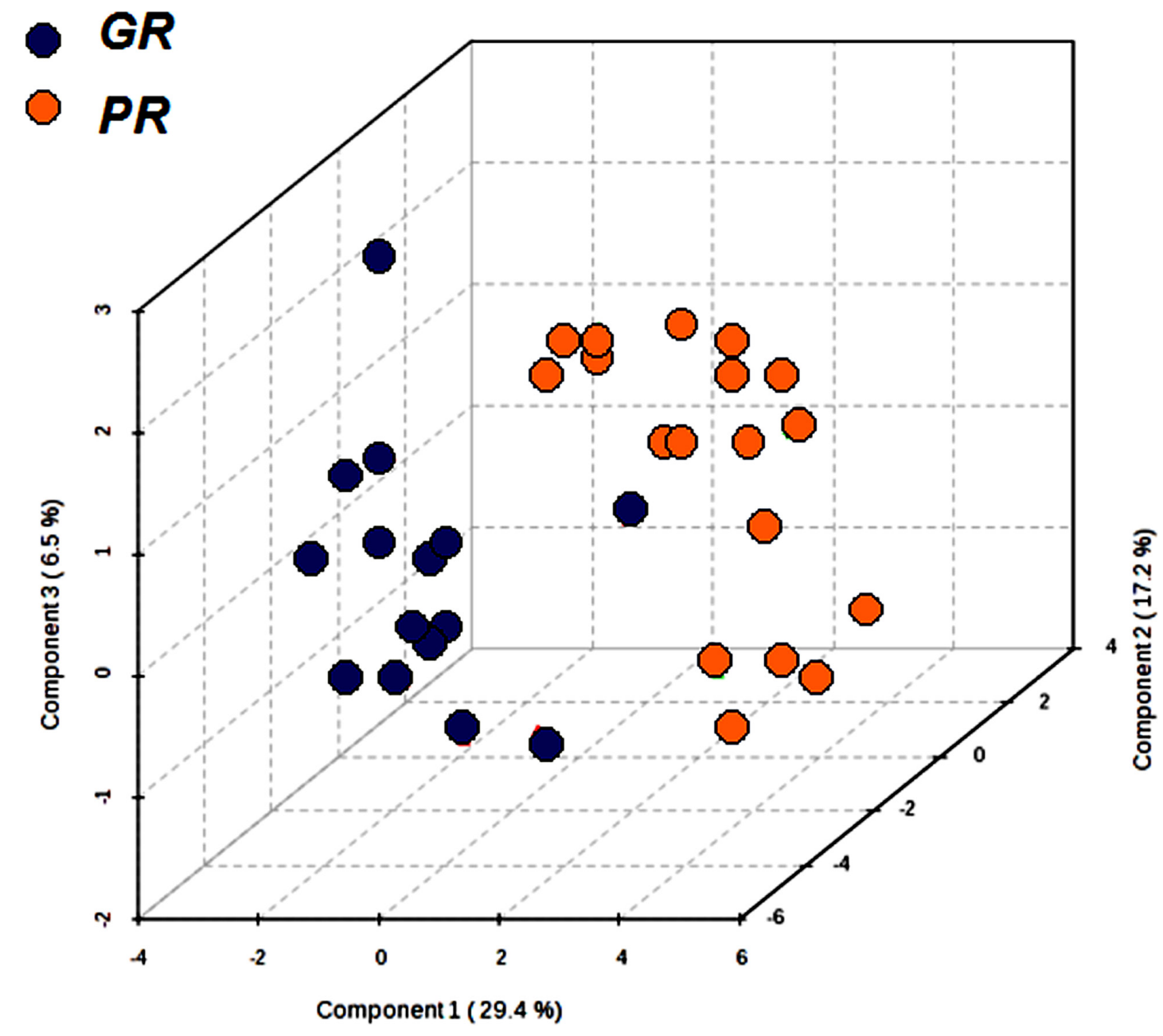

Figure 1: Partial least squares discrimination analysis (PLS-DA) graph used to distinguish the metabolomics profile of the two groups GR $(n=15)$ and PR $(n=19)$. Each point corresponds to the metabolomics profile of a patient. 
obtained by plotting the sensitivity and specificity values for each concentration of biomarkers and the threshold value for each metabolite was determined by searching for those that yielded both high sensitivity and specificity. The serum levels of the selected metabolic biomarkers Spd and Trp can be influenced by confounding effects such as age, BMI, and tumour stage. For this reason, the effect of these covariates on the sensitivity and specificity of ROC model, based on Trp and Spd metabolites, was investigated. The results of this investigation clearly showed that age, BMI and tumour stage had no effect on the predictive diagnostic power of Spd and Trp, indicating that these two metabolic biomarkers can be considered as independent factors for the response to neoadjuvant treatment (Figure S1 supplementary data). In Figure 7, the ROC curves for Spd, Trp, and Trp/Spd ratio with the associated area under curve (AUC) are reported. Considering Spd and Trp as single biomarkers, the AUC [95\% confidence interval] was 0.83 [0.67-0.95] and 0.87 [0.72-0.98] respectively, whilst the AUC value increased to $0.93[0.82-1.00]$ when the $\mathrm{Trp} / \mathrm{Spd}$ ratio was used. Therefore, using a threshold value of 565 for the Trp/Spd ratio it was possible to distinguish patients achieving a pCR after neoadjuvant treatment with high sensitivity 0.90 [0.79-1.00] and high specificity 0.87 [0.67-1.00].

\section{DISCUSSION}

In this pharmacometabolomics investigation, we applied a targeted metabolomics approach to assess the diagnostic potential of endogenous pre-treatment serum metabolites profile to predict the response to trastuzumabpaclitaxel treatment in a neoadjuvant setting. Unlike previous clinical metabolomics investigations [26, 27] this study focused on a homogeneous population of $\mathrm{BC}$ patients. We have observed that patients with higher serum concentrations of Spd and lower concentrations of Trp would be more likely to benefit from trastuzumabpaclitaxel neoadjuvant treatment. The Spd, spermine, and Put are essential ubiquitary cellular polyamines essentially constituted of organic polycations having variable hydrocarbon chains and two or more primary amino groups [28]. These chemical features are responsible for their essential role in DNA and RNA metabolism, maintenance of chromatin structure, regulation of specific gene expression, ion channel modulation and membrane stability. The key step in the production of polyamines is regulated by Orn decarboxylase (ODC), which is required for the production of Put. Subsequently, propylaminotransferase catalyzes the transfer of Put of the aminopropyl

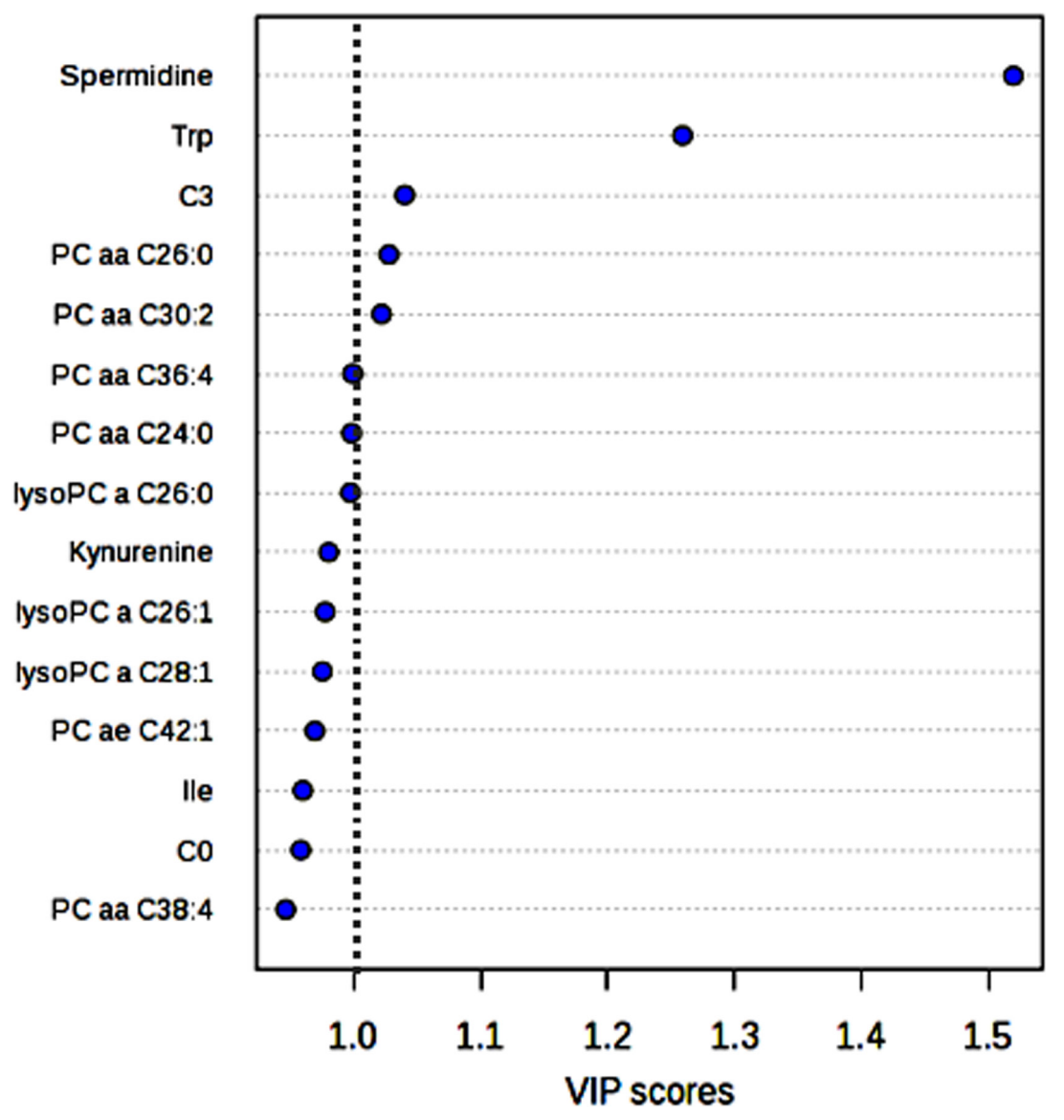

Figure 2: Metabolites that produce the largest contribution in discriminating between GR and PR groups in the PLS-DA model, relative to the VIP score. 
group from decarboxylated S-adenosylmethionine to form Spd and spermine, respectively (Figure 5). This process is important because polyamines are essential for growth and differentiation of cells. In general, high concentrations of polyamines are found in rapidly growing cells, while low concentrations are present in quiescent cells [29, 30]. An increased production is observed in many cancer tissues including BC [31]. However, the function of such high concentrations of polyamines in tumour cells is not clear. In a study addressed to evaluate the variation of polyamines in the pre and post-surgery serum of $\mathrm{BC}$ patients, no significant difference was reported [32]. This suggests that the circulatory level of polyamines is more likely to represent an individual's general physio-pathological condition. Thus, the difference in the Spd level between the GR and PR groups observed in this study could represent a host's metabolic feature not representative of the tumour's metabolic activity. In addition, the low level of serum Spd in the PR group appears not to be associated with differences in the level of Orn, Arg, Met and Put. Thus indicating that it may not be due to a shortage of the metabolite precursors. Moreover, the Orn/Spd and Put/Spd ratios, considered as surrogate parameters of Spd biosynthesis efficacy, were both lower in the GR group compared to PR patients. This suggests that a high synthetic rate of Spd could be a characteristic of patients that are more likely to achieve a pCR. However, a reduced catabolic clearance of Spd could also explain the higher concentration of Spd observed. Further investigation into the clearance of acetyl derivatives of polyamine [33] may give a more comprehensive explanation for the elevated Spd serum levels observed in the GR group.

It is possible that a high level of circulating Spd could be translated into a high concentration of polyamines in the tumour microenvironment that may boost cell replication, thus improving paclitaxel activity. This drug promotes the assembly of microtubules from tubulin dimers and stabilises them by preventing depolymerisation. This stability may cause the inhibition of the normal dynamic reorganisation of the microtubule network, which is essential for vital interphase and

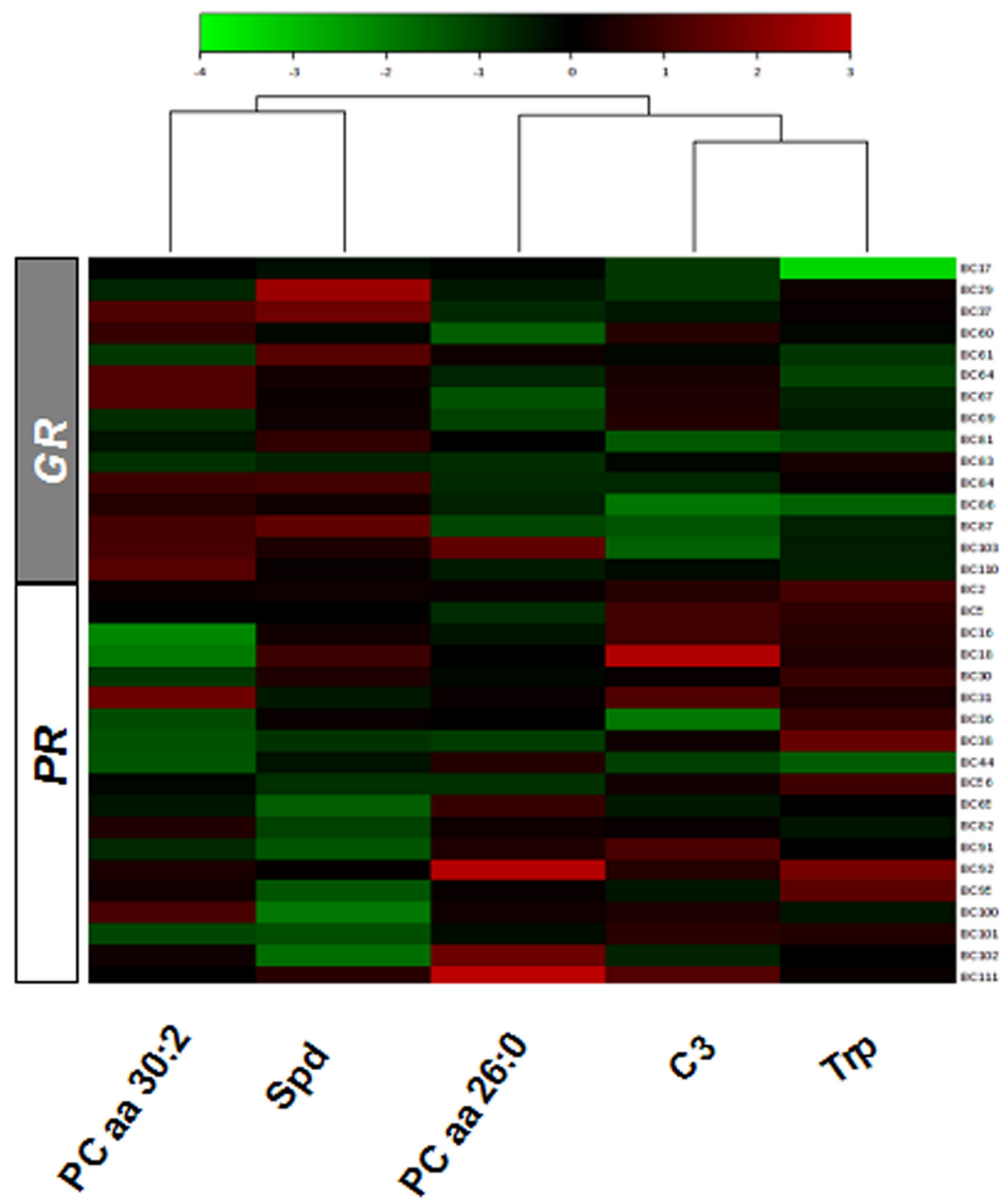

Figure 3: Heatmap of the relative concentration of five metabolites with VIP $>1$ in the serum of the GR and PR groups of patients according to the response to trastuzumab-paclitaxel treatment. 
mitotic cellular functions. Since Spd has been reported to improve tubulin assembly [34], a high concentration of this polyamine may also have a synergistic effect on the stabilization of microtubules, thus potentially increasing paclitaxel cytotoxicity.

The other relevant metabolic features that characterised the GR group of patients was the lower level of Trp. This metabolite is required for both protein synthesis and other important metabolic functions [35]. Low Trp serum levels may depend on a reduced dietary intake or an increase in its catabolism. Two major nonprotein metabolic routes use Trp as a precursor: $(a)$ the methoxyindoles pathway, involved in the synthesis of Set and Melatonin; and (b), the Kyn pathway, responsible for the production of Kyn through Niacin synthesis. Low levels of Trp, due to an increase activity of the Kyn pathway, can suppress the immune system [36-38]. Furthermore, the induction of this pathway has been recently proposed as a potential mechanism activated by the tumour to escape immune surveillance $[39 ; 40]$. Therefore, the involvement of Trp in the modulation of the immune system could be relevant for the immune- mediated activity of trastuzumab. Trastuzumab confers cytostatic effects through direct interaction with HER-2 receptors expressed by cancer cells suppressing the HER-2 dependent signalling pathway [41]. However, other mechanisms of action involving immune cells have been proposed for trastuzumab: the antibody-dependent cellular cytotoxicity (ADCC), which involves especially Natural Killer cells, and the activation of HER-2-specific cytotoxic T-cells $\left(\mathrm{CD}^{+}\right)$[14, 42]. Thus, the level of tryptophan and its catabolic product in the tumour microenvironment may contribute to modulate the immunocytoxic side activity induced by Trastuzumab.

When the levels of Kyn and Set were compared between the two groups, the GR showed lower levels of Kyn, suggesting that the lower amount of Trp observed was not due to an increased catabolism. Both groups had the same Kyn catabolic activity as demonstrated by the lack of significant differences in the Trp/Kyn ratio. Conversely, an increase in Trp anabolism via the Set pathway could also be potentially relevant in the PR group as reported by the lower Trp/Set ratio observed. The low availability of Trp may be associated with a

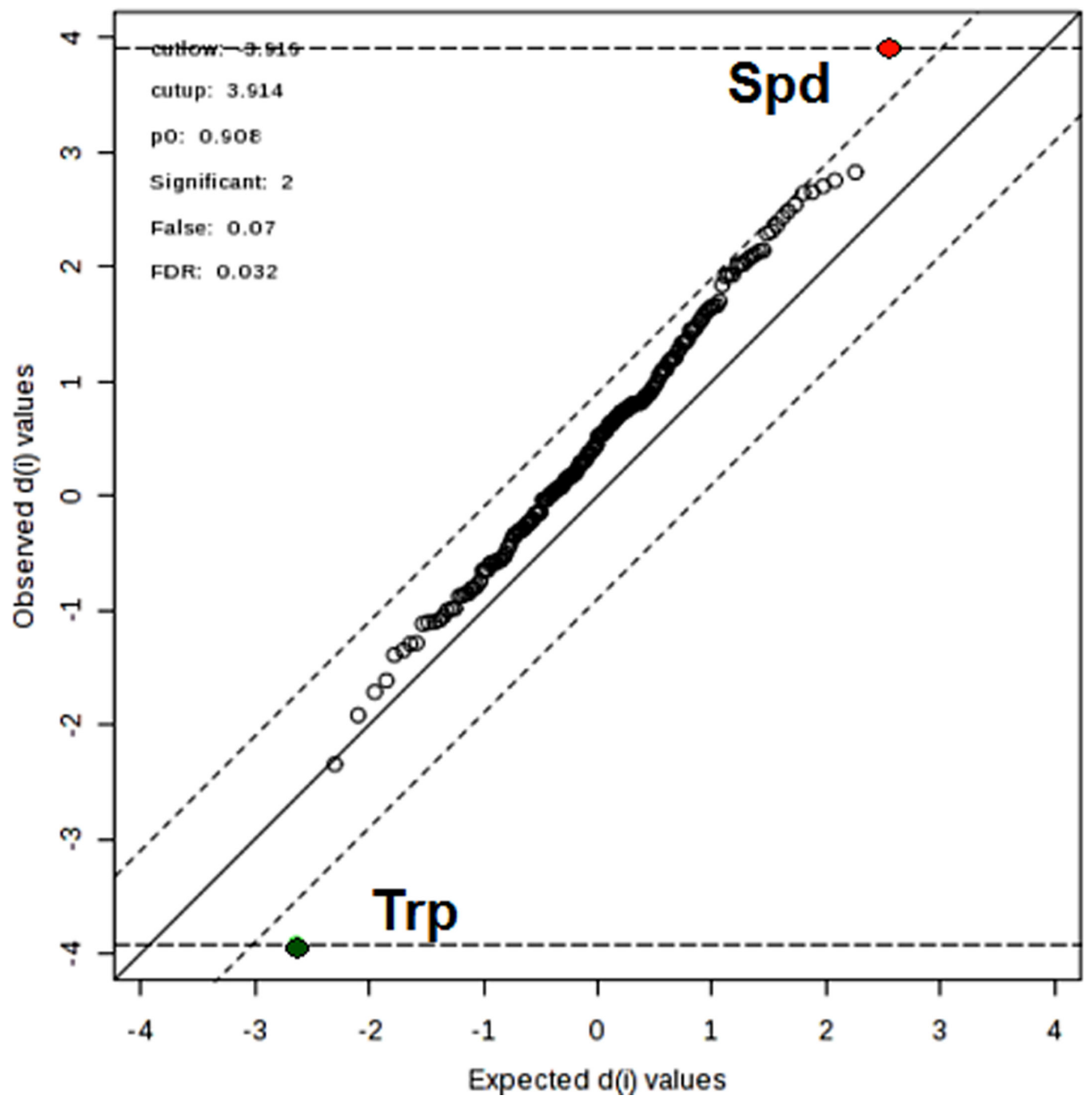

Figure 4: Significance analysis of microarray plot sheet output (SAM) under the four sets of criteria: cutup = 3.916, cutlow $=3.914$; false=0.07; FDR $=\mathbf{0 . 0 3 2}$; which are indicated at the upper right corner. The red, green, and black dots represent upregulated, downregulated, and insignificant metabolites. 


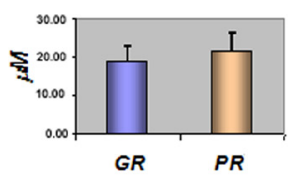

Methionine
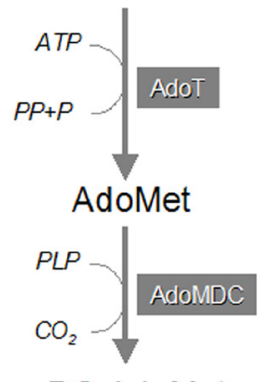

DC-AdoMet

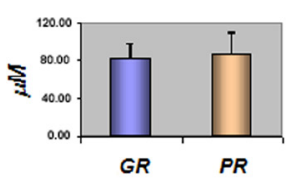

Ornitine
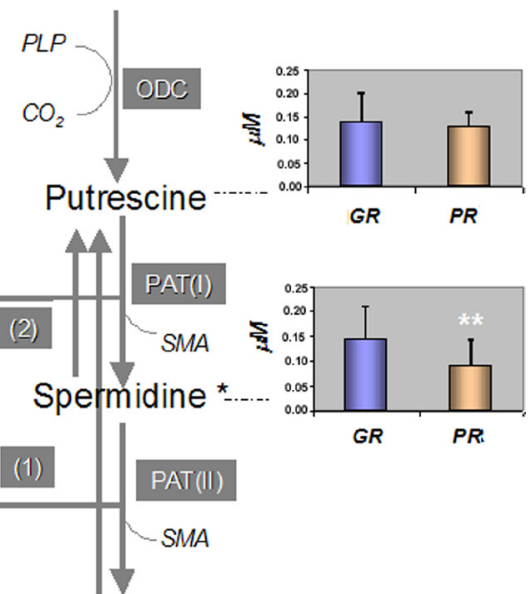

Spermine *

Figure 5: Polyamine biosyntethic pathway and the mean concentration $[\mu \mathrm{M}]$ of metabolites among the GR and PR groups of patients. A $p$ value of $* p<0.05$ and ${ }^{* *} p<0.001$ was considered statistically significant. AdoMet: S-Adenosyl-Methionine, DC-AdoMet: Decarboxylated S-Adenosyl-Methionine, AdoT: S-AdenosylTransferase, AdoMDC: S-Adenosyl-Methionine Decarboxylase, ODC: Ornitine Decarboxylase, PAT(I and II): PropylAminoTransferase-1 and 2, ATP: AdenosylTriphosphate. SMA: S-MethylAdenosyl. PLP: Pyridoxal-5'-Phosphate, (*) Spermine and Spermidine can be inter converted to Putrescine in two steps catalized by the (1) Spermine/ Spermidine-N-1-AcetylTransferase (SSAT); (2) Polyamine Oxidase (PO).

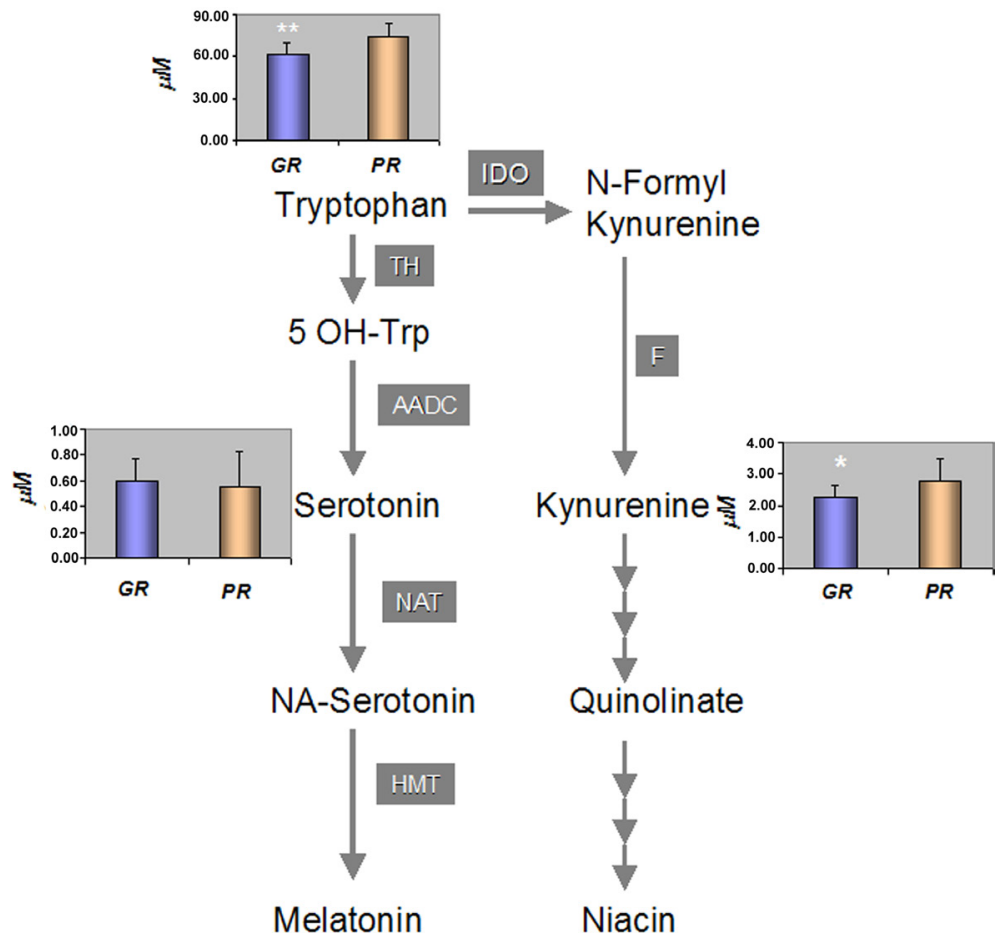

Figure 6: Tryptophan catabolic pathway and the mean concentration $[\mu \mathrm{M}]$ of metabolites among the GR and PR groups of patients. A $p$ value of $* p<0.05$ and ${ }^{*} p<0.001$ was considered statistically significant. TH: Tryptophan Hydroxylase, AADC: Aromatic Amino Acid Decarboxylase, NAT: N-Acetyl Transferase, HMT: 5-Hydroxylindole-O-MethylTransferase, Indoleamine-2, 3 dioxygenase, F: Formamidase. 
reduced Kyn biosynthesis, which acts as inhibitor of the immune response and thus may have a positive impact on the trastuzumab activity, contributing to the induction of a pCR in the GR group.

Finally and more importantly, the serum pretreatment levels of both Spd and Trp can be used as predictive markers to distinguish patients who are more likely to achieve pCR after trastuzumab-paclitaxel neoadjuvant treatment. In fact, when the two metabolites were combined in the Trp/Spd ratio, the inter-patient variation was reduced and the diagnostic power reached a high sensitivity of $90 \%$ with a specificity of $87 \%$ (Figure 7 ).

In conclusion, this exploratory study demonstrated the importance of highlighting endogenous metabolic pathways that could favour the efficacy of neoadjuvant trastuzumab-paclitaxel treatment in HER-2 positive BC patients. The pharmacometabolomics approach adopted identified two major metabolic biomarkers represented by, Spd and Trp, that could potentially have a pivotal role in determining the efficacy of a trastuzumab-paclitaxel drug combination. Such an individual metabolic signature could be translated into a non-invasive diagnostic tool to select
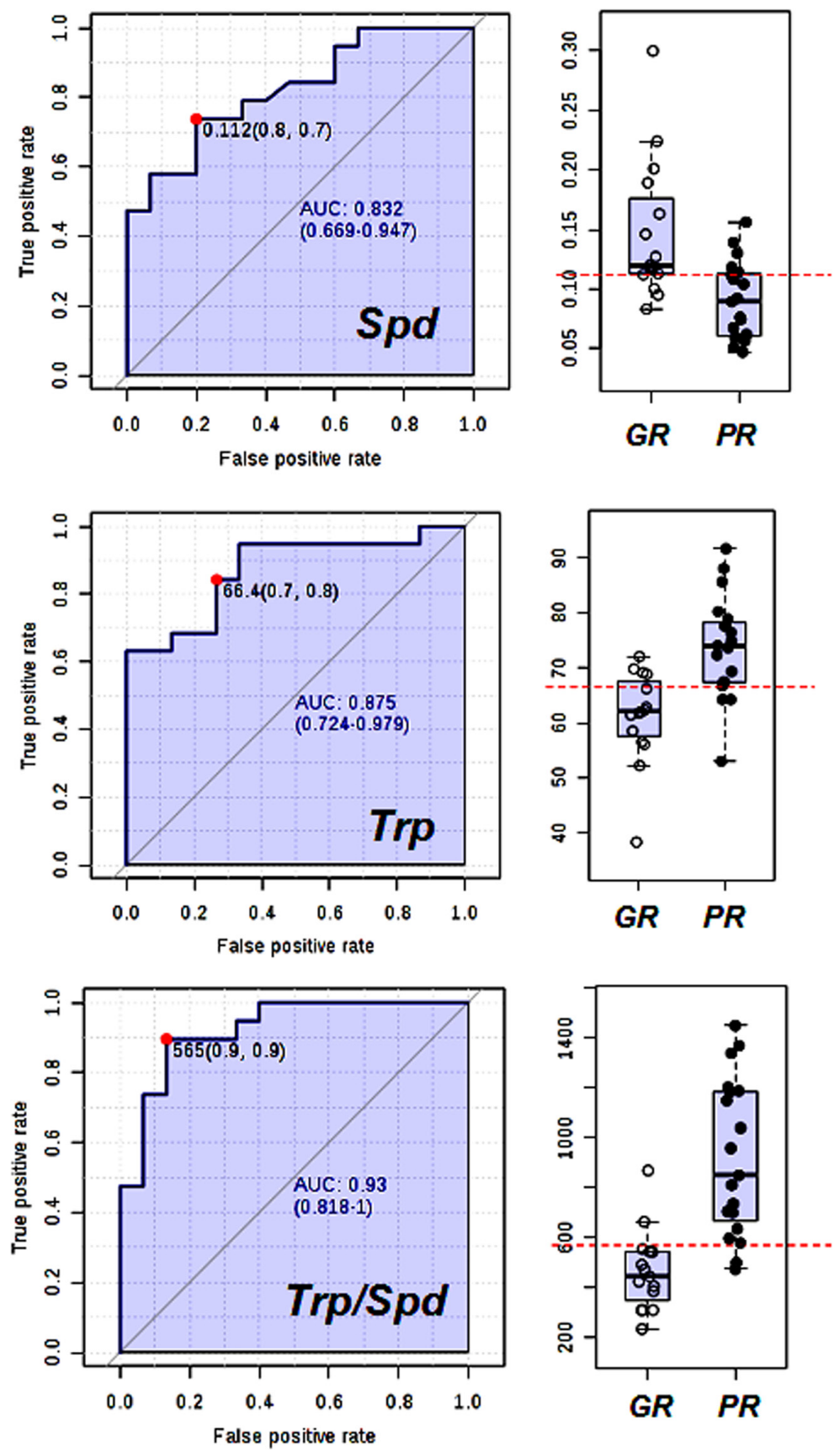

Figure 7: Receiving operating and scatter plots curve for (a) Spd, (b) Trp and (c) Trp/Spd ratio. Area under curve plots ( $95 \%$ confidence interval in brackets). 
patients more likely to benefit from neoadjuvant treatment. Although these preliminary results are very encouraging, they are based upon a relatively small sample size and additional studies with an increased number of patients will be needed to confirm and clinically validate these exciting observations.

\section{MATERIALS AND METHODS}

\section{Patients' population}

Thirty-four patients were enrolled in this investigation. All patients had histologically confirmed locally advanced HER-2 positive BC (UICC stage II-III, non-inflammatory); patients were not previously treated with chemotherapy or hormonal therapy, an age $\leq 70$ years, an Eastern Cooperative Oncology Group performance status $\leq 1$, adequate hepatic, renal and bone marrow function. No CNS metastases, prior myocardial infarction or uncontrolled arrhythmia, angina pectoris, psychiatric syndromes or other serious medical conditions were present. Furthermore, no other concurrent malignancy, apart from non-melanoma skin cancer, or in situ cervix carcinoma was allowed.

Baseline evaluation included a physical examination, diagnostic breast imaging, abdominal ultrasound and bone scintigraphy. This study was carried out according to the ethical principles of the Declaration of Helsinki and approved by the Internal Institutional ethics committee. All patients enrolled in the investigation gave written informed consent.

\section{Clinical samples}

After a period of overnight fasting, each patient provided a $5 \mathrm{ml}$ blood sample on the first day of admission, prior to receiving the neoadjuvant therapy. The blood was drawn in the morning between 8 and 10 a.m. to avoid variation due to circadian rhythms. Blood was collected in BD Vacutainer ${ }^{\circledR}$ tubes coated with silica particles as a clot activator. The blood was inverted five times and allowed 30 minutes clotting time and was then centrifuged at $2000 \mathrm{~g}$ for $10 \mathrm{~min}$ at $4^{\circ} \mathrm{C}$. Serum was then aliquot and frozen at $-80^{\circ} \mathrm{C}$ until required for targeted metabolomics analysis.

\section{Study design}

All patients enrolled in this study received trastuzumab: loading dose $4 \mathrm{mg} / \mathrm{kg}$ intravenously, then $2 \mathrm{mg} / \mathrm{kg}$ weekly and concomitant weekly paclitaxel $\left(80 \mathrm{mg} / \mathrm{m}^{2}\right)$ for 6 cycles. After neoadjuvant completion, patients underwent primary surgery, which consisted in a mastectomy or a quadrantectomy conservative treatment as well as axillary node dissection. In accordance to the histological response, the patients were classified in two groups: Good and Poor responders. The good responders (GR group) were patients who benefited from the treatment by achieving pCR corresponding to the histological disappearance of invasive cancer cells on breast tissue after surgery. Whilst patients who, after the neoadjuvant treatment, continued to show the presence of cancer cells in breast tissue were classified as poor responders (PR group).

The research of pre-treatment biomarkers predictive of the histological response was performed by analysing the quantitative differences in the serum targeted metabolomics profile among GR and PR group in a casecontrol study.

\section{Serum targeted metabolomics investigations}

A high-throughput liquid chromatography tandem mass spectrometry (LC-MS/MS) platform has been applied to evaluate the serum metabolomics profile using the AbsoluteIDQ p180 Kit (Biocrates Life Sciences Innsbruck, Austria). The analytical system consisted of a liquid chromatography ultimate 3000 (Thermo Fisher Scientific Milan Italy) coupled with a 4000 Qtrap (ABsciex Framingham, MA, USA) mass spectrometer. The analytical method allows the quantification of 188 targeted metabolites covering the following compound classes: amino acids, biogenic amines and polyamines ( $n=40)$, acylcarnitine $(n=40)$, di-acyl-phosphatidyl lipids $(n=92)$, sphingolipids $(n=15)$ and total exoses $(n=1)$. The complete list of the all metabolites investigated was reported in Table 2. Briefly, $10 \mu \mathrm{L}$ of serum were loaded onto a inserted filter in a 96 well sandwich plate, which already contained appropriate internal standards structurally identical but labeled with stable isotopes such as deuterium, $13 \mathrm{C}$, or $15 \mathrm{~N}$. The filters were dried in nitrogen stream, amino acids were derivatized with $5 \%$ phenylisothiocyanate reagent (PTC), and filters were dried again. After extraction of metabolites with $500 \mu \mathrm{L}$ of $5 \mathrm{mM}$ ammonium acetate in methanol, the solution was centrifuged through the filter membrane and diluted with water and MS running solvent. Final extracts were than analyzed by LC-MS/MS for amino acids and bioactive amines PTC-derivatives using a Zorbax SB $100 \times 2.1 \mathrm{~mm}$ column (Agilent, Santa Clara CA, USA), while direct flow injection analysis (FIA-MS/MS) was used for the analysis of acylcarnitine and phospolipides. The specific signals of metabolites utilized for quantitation were achieved by multiple reaction monitoring, neutral loss and precursor ion scans in positive and negative ion mode. The MS/MS signals were integrated, by using Analyst 1.6.1(ABsciex Framingham, MA, USA) and concentrations obtained using a calibration curve according to the AS-180 manual. All quantitative data were further processed for verification and validation by the MetIQ software by comparing the results of triplicate analysis of low, medium and high quality serum controls as an integral part of the analytical 
Table 2: List of metabolite determined using LC and FIA MS/MS platform

\begin{tabular}{|c|c|c|c|}
\hline Metabolite & $N$ & & \\
\hline Amino acids & 21 & $\begin{array}{l}\text { Ala, Arg, Asn, Asp, Cit, Gln, Glu, Gly, His, Ile, Leu, Lys, Met, } \\
\text { Orn, Phe, Pro, Ser, Thr, Trp, Tyr, Val }\end{array}$ & $\begin{array}{l}\text { Amino acid metabolism, urea-cycle, activity of } \\
\text { gluconeogenesis } \\
\text { and glycolysis, insulin sensitivity, neurotransmitter } \\
\text { metabolism, } \\
\text { oxidative stress }\end{array}$ \\
\hline Biogenic-amine & 19 & 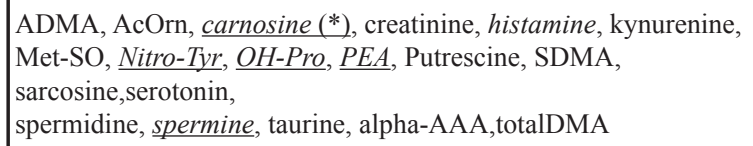 & $\begin{array}{l}\text { Neurological disorders, cell proliferation, cell cycle } \\
\text { progression, DNA stability, } \\
\text { oxidative stress }\end{array}$ \\
\hline Carnitine & 1 & $\mathrm{CO}$ & $\begin{array}{l}\text { Energy metabolism, fatty acid transport and } \\
\text { mitochondrial } \\
\text { fatty acid oxidation, ketosis, oxidative stress, } \\
\text { mitochondrial } \\
\text { membrane damage }\end{array}$ \\
\hline Acylcarnitines & 26 & $\begin{array}{l}\mathrm{C} 2, \mathrm{C} 3, \mathrm{C} 3: 1, \mathrm{C} 4, \mathrm{C} 4: 1, \mathrm{C} 5, \mathrm{C} 5: 1, \mathrm{C} 6 \text { (or C4:1-DC), } \mathrm{C} 6: 1, \mathrm{C} 8, \\
\mathrm{C} 8: 1, \underline{\mathrm{C} 9}, \mathrm{C} 10, \underline{\mathrm{C} 10: 1}, \mathrm{C} 10: 2, \mathrm{C} 12, \mathrm{C} 12: 1, \mathrm{C} 14, \mathrm{C} 14: 1, \mathrm{C} 14: 2, \\
\mathrm{C} 16, \underline{\mathrm{C} 16: 1}, \underline{\mathrm{C} 16: 2}, \underline{\mathrm{C} 18}, \mathrm{C} 18: 1, \mathrm{C} 18: 2\end{array}$ & “ \\
\hline $\begin{array}{l}\text { Hydroxy- and } \\
\text { dicarboxyacylcarnitines }\end{array}$ & 13 & $\begin{array}{l}\text { C3-OH, C4-OH (or C3-DC), C5-DC (or C6-OH), C5-OH(or } \\
\text { C3-DC-M), C5:1-DC, C5-M-DC, C7-DC, C12-DC, C14:1-OH, } \\
\text { C14:2-OH, C16:1-OH, C16:2-OH, C16-OH,C18:1-OH }\end{array}$ & “ \\
\hline Sum of hexoses & 1 & H1 & Carbohydrate metabolism \\
\hline Sphingomyelins & 10 & $\begin{array}{l}\text { SM C16:0, SM C16:1, SM C18:0, SM C18:1, SMC20:2, SM } \\
\text { C22:3, SM C24:0, SMC24:1, SM C26:0,SM C26:1 }\end{array}$ & $\begin{array}{l}\text { Signalling cascades, membrane damage } \\
\text { (eg,neurodegeneratio)n }\end{array}$ \\
\hline Hydroxysphingomyelins & 5 & $\begin{array}{l}\mathrm{SM}(\mathrm{OH}) \mathrm{C} 14: 1, \mathrm{SM}(\mathrm{OH}) \mathrm{C} 16: 1, \mathrm{SM}(\mathrm{OH}) \mathrm{C} 22: 1, \mathrm{SM}(\mathrm{OH}) \\
\mathrm{C} 22: 2, \mathrm{SM}(\mathrm{OH}) \mathrm{C} 24: 1\end{array}$ & “ \\
\hline $\begin{array}{l}\text { Diacyl- } \\
\text { phosphatidylcholines }\end{array}$ & 38 & $\begin{array}{l}\mathrm{PC} \text { aa C24:0/C26:0/C28:1/C30:0/C30:2/C32:0/C32:1/C32:2/ } \\
\mathrm{C} 32: 3 / \mathrm{C} 34: 1 / \mathrm{C} 34: 2 / \mathrm{C} 34: 3 / \mathrm{C} 34: 4 / \mathrm{C} 36: 0 / \mathrm{C} 36: 1 / \mathrm{C} 36: 2 / \mathrm{C} 36: 3 / \\
\mathrm{C} 36: 4 / \mathrm{C} 36: 5 / \mathrm{C} 36: 6 / \mathrm{C} 38: 0 \mathrm{C} 38: 1 / \mathrm{C} 38: 3 / \mathrm{C} 38: 4 / \mathrm{C} 38: 5 / \mathrm{C} 38: 6 / \\
\mathrm{C} 40: 1 / \mathrm{C} 40: 2 \mathrm{C} 40: 3 / \mathrm{C} 40: 4 / \mathrm{C} 40: 5 / \mathrm{C} 40: 6 / \mathrm{C} 42: 0 / \mathrm{C} 42: 1 / \mathrm{C} 42: 2 / \\
\mathrm{C} 42: 4 / \mathrm{C} 42: 5 / \mathrm{C} 42: 6\end{array}$ & $\begin{array}{l}\text { Degradation of phospholipids, membrane damage, } \\
\text { signalling } \\
\text { cascades, fatty acid profile }\end{array}$ \\
\hline $\begin{array}{l}\text { Acyl-alkyl- } \\
\text { phosphatidylcholines }\end{array}$ & 39 & $\begin{array}{l}\mathrm{PC} \text { ae } \\
\mathrm{C} 30: 0 / \mathrm{C} 30: 1 / \mathrm{C} 30: 2 / \mathrm{C} 32: 1 / \mathrm{C} 32: 2 / \mathrm{C} 34: 0 / \mathrm{C} 34: 1 / \mathrm{C} 34: 2 / \mathrm{C} 34: 3 / \\
\mathrm{C} 36: 0 / \mathrm{C} 36: 1 / \mathrm{C} 36: 2 / \mathrm{C} 36: 3 / \mathrm{C} 36: 4 / \mathrm{C} 36: 5 / \mathrm{C} 38: 0 / \mathrm{C} 38: 1 / \mathrm{C} 38: 2 / \\
\mathrm{C} 38: 3 / \mathrm{C} 38: 4 / \mathrm{C} 38: 5 / \mathrm{C} 38: 6 / \mathrm{C} 40: 0 / \mathrm{C} 40: 1 / \mathrm{C} 40: 2 / \mathrm{C} 40: 3 / \mathrm{C} 40: 4 / \\
\mathrm{C} 40: 5 / \mathrm{C} 40: 6 / \mathrm{C} 42: 0 / \mathrm{C} 42: 1 / \mathrm{C} 42: 2 / \mathrm{C} 42: 3 / \mathrm{C} 42: 4 / \mathrm{C} 42: 5 / \mathrm{C} 44: 3 / \\
\mathrm{C} 44: 4 / \mathrm{C} 44: 5 / \mathrm{C} 44: 6\end{array}$ & “ \\
\hline $\begin{array}{l}\text { Lyso- } \\
\text { phosphatidylcholines }\end{array}$ & 15 & $\begin{array}{l}\text { lysoPC aa C6:0/C14:0/C16:0/C16:1/C17:0/C18:0/C18:1/C18:2/ } \\
\text { C20:3/C20:4/C24:0/C26:0/C26:1/C28:0/C28:1 }\end{array}$ & $\begin{array}{l}\text { Degradation of phospholipids (phospholipase } \\
\text { activity), membrane damage, signaling cascades, } \\
\text { fatty acid profiles }\end{array}$ \\
\hline
\end{tabular}

aa, acyl-acyl; ae, acyl-alkyl; a, lyso; Cx:y, where $\mathrm{x}$ is the number of carbons in the fatty acid side chain; $\mathrm{y}$ is the number of double bonds in the fatty acid side chain; DC, decarboxyl; M,methyl; OH, hydroxyl; PC, phophatidylcholine; SM, sphingomyelin. $(*)$ Underlined the metabolites that did not pass the analytical quality control process.

kit. Within the analytical batch the coefficient of variation for the quality control metabolites concentration was less than $20 \%$, while the uncertainty of the measurements was found in the range of $80-115 \%$ of their theoretical values. Samples were analyzed in a random sequence with serum controls in compliance with Want et al. 2010 guidelines [43]. The metabolites with serum concentration above the limit of detection in at least $50 \%$ of all measured samples were excluded by successive statistical analysis. Among the serum samples investigated, about $18 \%$ of analyzed metabolites showed serum concentrations below the lower limit of quantification criteria and were not considered for further data analysis. The final number of metabolites analyzed was $153(82 \%)$. This set included $17(42 \%)$ acylcarnitines, 14 (100\%) lysophosphatidyl coline lipids, 72 (95\%) phosphatidylcolines, 15 (100\%) sphingomyelin lipids and 35 (83\%) amino acids plus bioactive amine derivatives. The mean \pm SD serum concentration of the validated metabolites among the GR and PR groups of patients are reported together with the statistical significance in supplemental data in Table S1

\section{Data processing and statistical analysis}

Prior to statistical analysis, the concentration values of metabolites were set to a log scale and auto- 
scaled (mean-centred and divided by the standard deviation of each variable). A supervisor multivariate partial least squares discrimination analysis (PLS-DA) was then applied to identify the relevant metabolites that contribute the most significantly in differentiating between the two groups of patients. The PLS model was refined by incorporating only the metabolite variable that was identified as being significantly different among the two groups of patients $(p<0.05)$. A variable importance in projection (VIP) score was also applied to rank the patient's metabolites that best distinguished between the GR $v s$ PR group. The PSA-DA model was further cross-validated by comparison of the resulting goodness of fit $\left(R^{2}\right)$, predictive ability $\left(Q^{2}\right)$ values, and by internal validation using 1000 permutation tests. $Q^{2}$ represents the most recognized diagnostic statistical parameter to validate the PLS-DA model in metabolomics. It is calculated as 1-PRESS/TSS where PRESS is the sum of squared differences between the predicted and real value, while the TSS is the total sum of the square. Significant predictive model is considered for $Q^{2}$ value greater than 0.4 . $R^{2}$ indicates the total variation in the data matrix that is explained by the model. It is calculated as 1- RSS/TSS where the RSS is the fitted residual sum of the square of the data. An invalid or irrelevant model may be still capable of producing a large $Q^{2}$ value, because consistent cross-validation requires a systematic deletion of large portions of the dataset during training. Thus, further validation of the model was performed by random 1000 permutation of class labels, which requires no deletion of data, with internal leave-n-out cross-validation. Significance of permutation test was expressed as pseudo $\mathrm{p}$ calculated as number of failed prediction / numbers of permutation performed.

The significant features were further selected by taking into account the problem of false discovery rate (FDR) occurring for multiple analyses [44], through the application of the significance analysis of microarray (SAM) approach. An un-paired $t$-test was used as a univariate analysis to check the differences in the serum concentrations of single metabolites between the GR and the PR groups. For all the above univariate tests $q$ values were calculated to control for multiple testing false discovery rate.

Receiver operating characteristic (ROC) curves analysis was used to determine the diagnostic power of the more significant metabolomics biomarkers. In a ROC curve, the true positive rate (sensitivity) was plotted against the false positive rate (1-specificity) for different cut-off points of a given parameter. The ROC curve was validated by Internal cross validation and permutation testing. The optimal cut-off was assessed by jointly maximizing sensitivity and specificity. Sensitivity and specificity, computed at the optimal cut-off, were then used for further investigation. All above data processing and the statistical analysis that included ROC analysis was performed using the Metabolanalyst web portals [45, 46].

\section{ACKNOWLEDGMENTS AND FUNDING}

This research study was supported by grant from "FONDAZIONE UMBERTO VERONESI" (G.C.) and My FIRST AIRC No. 1569 (F.R.). EM was supported by a FONDAZIONE UMBERTO VERONESI Fellowship. We thank Prof. Eugenio Ragazzi for the useful discussion and suggestions.

\section{CONFLICTS OF INTEREST}

There are no conflicts of interests to declare.

\section{REFERENCES}

1. Slamon DJ, Leyland-Jones B, Shak S, Fuchs H, Paton V, Bajamonde A, Fleming T, Eiermann W, Wolter J, Pegram M, Baselga J, Norton L. Use of chemotherapy plus a monoclonal antibody against HER2 for metastatic breast cancer that overexpresses HER2. N Engl J Med. 2001; 344:783-792.

2. Goldhirsch A, Gelber RD, Piccart-Gebhart MJ, de AE, Procter M, Suter TM, Jackisch C, Cameron D, Weber HA, Heinzmann D, Dal LL, McFadden E, Dowsett M, et al. 2 years versus 1 year of adjuvant trastuzumab for HER2positive breast cancer (HERA): an open-label, randomised controlled trial. Lancet. 2013; 382:1021-1028.

3. Smith I, Procter M, Gelber RD, Guillaume S, Feyereislova A, Dowsett M, Goldhirsch A, Untch M, Mariani G, Baselga J, Kaufmann M, Cameron D, Bell R, et al. 2-year follow-up of trastuzumab after adjuvant chemotherapy in HER2-positive breast cancer: a randomised controlled trial. Lancet. 2007; 369:29-36.

4. Piccart-Gebhart MJ, Procter M, Leyland-Jones B, Goldhirsch A, Untch M, Smith I, Gianni L, Baselga J, Bell R, Jackisch C, Cameron D, Dowsett M, Barrios CH, et al. Trastuzumab after adjuvant chemotherapy in HER2-positive breast cancer. N Engl J Med. 2005; 353:1659-1672.

5. Gianni L, Eiermann W, Semiglazov V, Lluch A, Tjulandin S, Zambetti M, Moliterni A, Vazquez F, Byakhov MJ, Lichinitser M, Climent MA, Ciruelos E, Ojeda B, et al. Neoadjuvant and adjuvant trastuzumab in patients with HER2-positive locally advanced breast cancer (NOAH): follow-up of a randomised controlled superiority trial with a parallel HER2-negative cohort. Lancet Oncol. 2014; 15:640-647.

6. von MG, Untch M, Blohmer JU, Costa SD, Eidtmann H, Fasching PA, Gerber B, Eiermann W, Hilfrich J, Huober J, Jackisch C, Kaufmann M, Konecny GE, et al. Definition and impact of pathologic complete response on prognosis after neoadjuvant chemotherapy in various intrinsic breast cancer subtypes. J Clin Oncol. 2012; 30:1796-1804.

7. Kim MM, Allen P, Gonzalez-Angulo AM, Woodward WA, Meric-Bernstam F, Buzdar AU, Hunt KK, Kuerer HM, Litton JK, Hortobagyi GN, Buchholz TA, Mittendorf EA. Pathologic complete response to neoadjuvant chemotherapy 
with trastuzumab predicts for improved survival in women with HER2-overexpressing breast cancer. Ann Oncol. 2013; 24:1999-2004.

8. Buzdar AU, Valero V, Ibrahim NK, Francis D, Broglio KR, Theriault RL, Pusztai L, Green MC, Singletary SE, Hunt KK, Sahin AA, Esteva F, Symmans WF, et al. Neoadjuvant therapy with paclitaxel followed by 5-fluorouracil, epirubicin, and cyclophosphamide chemotherapy and concurrent trastuzumab in human epidermal growth factor receptor 2-positive operable breast cancer: an update of the initial randomized study population and data of additional patients treated with the same regimen. Clin Cancer Res. 2007; 13:228-233.

9. Houssami N, Macaskill P, von MG, Marinovich ML, Mamounas E. Meta-analysis of the association of breast cancer subtype and pathologic complete response to neoadjuvant chemotherapy. Eur J Cancer. 2012; 48:3342-3354.

10. Nahta R, Shabaya S, Ozbay T, Rowe DL. Personalizing HER2-targeted therapy in metastatic breast cancer beyond HER2 status: what we have learned from clinical specimens. Curr Pharmacogenomics Person Med. 2009; 7:263-274.

11. Krop IE, Burstein HJ. Trastuzumab. qui bono? J Natl Cancer Inst. 2013; 105:1772-1775.

12. Pogue-Geile KL, Kim C, Jeong JH, Tanaka N, Bandos H, Gavin PG, Fumagalli D, Goldstein LC, Sneige N, Burandt E, Taniyama Y, Bohn OL, Lee A, et al. Predicting degree of benefit from adjuvant trastuzumab in NSABP trial B-31. J Natl Cancer Inst .2013; 105:1782-1788.

13. De P, Hasmann M, Leyland-Jones B. Molecular determinants of trastuzumab efficacy: What is their clinical relevance? Cancer Treat Rev. 2013; 39:925-934.

14. Arnould L, Gelly M, Penault-Llorca F, Benoit L, Bonnetain F, Migeon C, Cabaret V, Fermeaux V, Bertheau P, Garnier J, Jeannin JF, Coudert B. Trastuzumab-based treatment of HER2-positive breast cancer: an antibody-dependent cellular cytotoxicity mechanism? Br J Cancer. 2006; 94:259-267.

15. Fontanella C, Lederer B, Gade S, Vanoppen M, Blohmer JU, Costa SD, Denkert C, Eidtmann H, Gerber B, Hanusch C, Hilfrich J, Huober J, Schneeweiss A, et al. Impact of body mass index on neoadjuvant treatment outcome: a pooled analysis of eight prospective neoadjuvant breast cancer trials. Breast Cancer Res Treat. 2015; 150:127-139.

16. Clayton TA, Lindon JC, Cloarec O, Antti H, Charuel C, Hanton G, Provost JP, Le Net JL, Baker D, Walley RJ, Everett JR, Nicholson JK. Pharmaco-metabonomic phenotyping and personalized drug treatment. Nature. 2006; 440:1073-1077.

17. Clayton TA, Baker D, Lindon JC, Everett JR, Nicholson JK. Pharmacometabonomic identification of a significant host-microbiome metabolic interaction affecting human drug metabolism. Proc Natl Acad Sci U S A. 2009; 106: 14728-14733.
18. Nicholson JK, Wilson ID, Lindon JC. Pharmacometabonomics as an effector for personalized medicine. Pharmacogenomics. 2011; 12:103-111.

19. Everett JR, Loo RL, Pullen FS. Pharmacometabonomics and personalized medicine. Ann Clin Biochem. 2013; 50:523-545.

20. Corona G, Rizzolio F, Giordano A, Toffoli G. Pharmacometabolomics: an emerging "omics" tool for the personalization of anticancer treatments and identification of new valuable therapeutic targets. J Cell Physiol. 2012; 227:2827-2831.

21. Kaddurah-Daouk R, Boyle SH, Matson W, Sharma S, Matson S, Zhu H, Bogdanov MB, Churchill E, Krishnan RR, Rush AJ, Pickering E, Delnomdedieu M. Pretreatment metabotype as a predictor of response to sertraline or placebo in depressed outpatients: a proof of concept. Transl Psychiatry. 2011; 1. pii: e26. doi: 10.1038/tp.2011.22.:e26.

22. Kaddurah-Daouk R, Weinshilboum RM. Pharmacometabolomics: implications for clinical pharmacology and systems pharmacology. Clin Pharmacol Ther. 2014; 95:154-167.

23. Krauss RM, Zhu H, Kaddurah-Daouk R. Pharmacometabolomics of statin response. Clin Pharmacol Ther. 2013; 94:562-565.

24. Phapale PB, Kim SD, Lee HW, Lim M, Kale DD, Kim YL, Cho JH, Hwang D, Yoon YR. An integrative approach for identifying a metabolic phenotype predictive of individualized pharmacokinetics of tacrolimus. Clin Pharmacol Ther. 2010; 87:426-436.

25. Zhu H, Bogdanov MB, Boyle SH, Matson W, Sharma S, Matson S, Churchill E, Fiehn O, Rush JA, Krishnan RR, Pickering E, Delnomdedieu M, Kaddurah-Daouk R. Pharmacometabolomics of response to sertraline and to placebo in major depressive disorder-possible role for methoxyindole pathway. PLoS One. 2013; 8:e68283.

26. Tenori L, Oakman C, Morris PG, Gralka E, Turner N, Cappadona S, Fornier M, Hudis C, Norton L, Luchinat C, Di LA. Serum metabolomic profiles evaluated after surgery may identify patients with oestrogen receptor negative early breast cancer at increased risk of disease recurrence. Results from a retrospective study. Mol Oncol. 2014; 10.

27. Wei S, Liu L, Zhang J, Bowers J, Gowda GA, Seeger H, Fehm T, Neubauer HJ, Vogel U, Clare SE, Raftery D. Metabolomics approach for predicting response to neoadjuvant chemotherapy for breast cancer. Mol Oncol. 2013; 7:297-307.

28. Ramani D, De Bandt JP, Cynober L. Aliphatic polyamines in physiology and diseases. Clin Nutr. 2014; 33:14-22.

29. Casero RA Jr, Wang Y, Stewart TM, Devereux W, Hacker A, Wang Y, Smith R, Woster PM. The role of polyamine catabolism in anti-tumour drug response. Biochem Soc Trans. 2003; 31:361-365. 
30. Nowotarski SL, Woster PM, Casero RA Jr. Polyamines and cancer: implications for chemotherapy and chemoprevention. Expert Rev Mol Med. 2013;15:e3. doi: 10.1017/erm.2013.3.:e3.

31. Leveque J, Bansard JY, Watier E, Catros-Quemener V, Havouis R, Moulinoux JP, Grall JY, Seiler N. Polyamines in human breast cancer and its relations to classical prognostic features: clinical implications. Anticancer Res. 1999; 19:2275-2279.

32. Byun JA, Choi MH, Moon MH, Kong G, Chul CB. Serum polyamines in pre- and post-operative patients with breast cancer corrected by menopausal status. Cancer Lett. 2009; 273:300-304.

33. Tsutsui H, Mochizuki T, Inoue K, Toyama T, Yoshimoto N, Endo Y, Todoroki K, Min JZ, Toyo'oka T. High-throughput LC-MS/MS based simultaneous determination of polyamines including $\mathrm{N}$-acetylated forms in human saliva and the diagnostic approach to breast cancer patients. Anal Chem. 2013; 85:11835-11842.

34. Mechulam A, Chernov KG, Mucher E, Hamon L, Curmi PA, Pastre D. Polyamine sharing between tubulin dimers favours microtubule nucleation and elongation via facilitated diffusion. PLoS Comput Biol. 2009; 5:e1000255.

35. Bender DA. Biochemistry of tryptophan in health and disease. Mol Aspects Med. 1983; 6:101-197.

36. Johnson TS, Munn DH. Host indoleamine 2, 3-dioxygenase: contribution to systemic acquired tumor tolerance. Immunol Invest 2012;41:765-797.

37. Mandi Y, Vecsei L. The kynurenine system and immunoregulation. J Neural Transm. 2012; 119:197-209.

38. Belladonna ML, Puccetti P, Orabona C, Fallarino F, Vacca C, Volpi C, Gizzi S, Pallotta MT, Fioretti MC, Grohmann U. Immunosuppression via tryptophan catabolism: the role of kynurenine pathway enzymes. Transplantation. 2007; 84:S17-S20.
39. Prendergast GC. Cancer. Why tumours eat tryptophan. Nature. 2011; 478:192-194.

40. Cascino A, Cangiano C, Ceci F, Franchi F, Mineo T, Mulieri M, Muscaritoli M, Rossi FF. Increased plasma free tryptophan levels in human cancer: a tumor related effect? Anticancer Res. 1991; 11:1313-1316.

41. Valabrega G, Montemurro F, Aglietta M. Trastuzumab: mechanism of action, resistance and future perspectives in HER2-overexpressing breast cancer. Ann Oncol. 2007; 18:977-984.

42. Kono K, Sato E, Naganuma H, Takahashi A, Mimura K, Nukui H, Fujii H. Trastuzumab (Herceptin) enhances class I-restricted antigen presentation recognized by HER-2/neuspecific T cytotoxic lymphocytes. Clin Cancer Res. 2004; 10:2538-2544.

43. Want EJ, Wilson ID, Gika H, Theodoridis G, Plumb RS, Shockcor J, Holmes E, Nicholson JK. Global metabolic profiling procedures for urine using UPLC-MS. Nat Protoc 20. 10; 5:1005-1018.

44. Benjamini Y, Hochberg Y. Controlling the false discovery rate: a practical and powerful approach to multiple testing. Journal of the Royal Statistical Society, Series B 1995; 57:289-300.

45. Xia J, Sinelnikov IV, Han B, Wishart DS. MetaboAnalyst 3.0-making metabolomics more meaningful. Nucleic Acids Res. 2015; 43:W251-W257.

46. Xia J, Broadhurst DI, Wilson M, Wishart DS: Translational biomarker discovery in clinical metabolomics: an introductory tutorial. Metabolomics. 2013; 9:280-299. 